\title{
Association of sericin and swimming on the phenotype, motor plate, and functionality of the denervated plantar muscle of Wistar rats
}

\author{
André Junior Santana', Jean Carlos Debastiani', Regina Inês Kunz², Pamela Buratti', Rose Meire Costa Brancalhão², \\ Lucinéia de Fátima Chasko Ribeiro², Márcia Miranda Torrejais², Gladson Ricardo Flor Bertolini²,** \\ 'Programa de Pós-Graduação em Biociências e Saúde da, Universidade Estadual do Oeste do Paraná (UNIOESTE), Cascavel, Brazil \\ ²universidade Estadual do Oeste do Paraná (UNIOESTE), Cascavel, Brazil
}

Physical exercise may help maintain muscle properties and functional improvement after peripheral nerve lesion, which may be enhanced by using biocompatible substances, such as sericin. The aim of this study was analyse the effect of sericin associated with swimming exercise on the phenotype, innervation, and functionality of the plantar muscle of Wistar rats. Forty randomly divided adult rats were used in five groups of eight animals: control, injury, sericin, exercise, exercise and sericin. The application of sericin was done on the spot, $100 \mu \mathrm{L}$, shortly after nerve compression. Three days after sciatic nerve compression, the swimming and swimming and sericin groups were submitted to physical swimming exercise for 21 days. Afterwards, the animals were euthanised and the plantar muscle was dissected and submitted to histochemical and histoenzymological techniques. The grip strength test did not show alterations in muscular functionality, and the control present-

\section{INTRODUCTION}

Peripheral nerve lesion (PNL) can result in a variety of sensory and autonomic implications, including paresis and plegia, leading to hypotrophies, as well as pain and oedema, paraesthesias and functional impairment, resulting from a communication deficit between nerve and muscle (da Silva and Camargo, 2010). In developed countries, the annual occurrence of lesions is estimated between 13 and 23 cases per 100,000 inhabitants, mainly affecting the economically active population, such as young people between 21 and 30 years old (Li et al., 2014). ed greater muscle mass in relation to the other groups, the same did not occur for muscle length. Polymorphic neuromuscular junctions were detected in the groups, although without significant morphometric alterations of the area, major and minor diameters. The percentage of type I fibres was lower in the lesion group and there was no difference in fibres Ila and Ilb between groups. The area of fibres I, Ila and Ilb remained constant between groups. Sericin biopolymer combined with swimming exercise did not affect plantar muscle function, submitted to experimental axonotmosis, whose contractile properties were altered by nerve injury.

Keywords: Peripheral nerves, Muscle fibre, Physical exercise, Nerve injury, Silk protein
${ }^{*}$ Corresponding author: Gladson Ricardo Flor Bertolini

(iD) https://orcid.org/0000-0003-0565-2019

Universidade Estadual do Oeste do Paraná (UNIOESTE), Rua Universitária, 2069, Cascavel 85819-110, Brazil

Tel: +55-45-3220-7343, Fax: +55-45-3220-3157

E-mail: gladsonricardo@gmail.com

Received: October 17, 2017 / Accepted: January 23, 2018
Within the experimental models of nerve compression, the sciatic nerve of rats is widely used because of its morphological characteristics, as well as easy surgical access and because it has previous data for comparison (Geuna, 2015). One of the characteristics of this nervous compression is weakness of the muscles of the pelvic limb, producing muscular denervation leading to atrophy, fibrosis, transient dilation of the intramuscular capillary bed, causing an increase in muscle blood volume and consequently an increase of extracellular fluid, in addition to increased proteolysis (Käriäinen and Kauhanen, 2012).

Neuromuscular junctions (NMJs) have three main structural 
elements: the presynaptic region, the synaptic cleft and the postsynaptic region (Gonzalez-Freire et al., 2014). NMJs are elements of the nervous system that play a key role in signal transmission between motor neurons and muscle fibres (Wu et al., 2010). The impairment of NMJ function results in muscle weakness or paralysis, impairing existing triggers that perform signalling in skeletal muscle, ultimately leading to severe muscle loss (Kwon and Yoon, 2017).

Studies show that both denervation and physical exercise modify the morphological properties of NMJs (Deschenes et al., 2011, Deschenes et al., 2015; Torrejais et al., 2009). Among the therapeutic strategies used in rehabilitation are physical swimming exercises that consist of activity aimed at improving muscular properties, through aerobic training, as well as phenotypic transformation of skeletal muscle (Yan et al., 2011). There are indications of the benefits of physical exercise in muscle regeneration, as in preventing atrophy and improving the structural properties of muscle. However, its effects on skeletal muscle, after compressive injury of the sciatic nerve, are much discussed, especially in relation to the type of exercise, its intensity and the best period in which to initiate it (Malysz et al., 2011; Tanaka et al., 2005).

Like physical exercise, the use of biocompatible materials, which may aid muscle recovery is relevant. Among these materials, the proteins fibroin and sericin extracted from the silkworm cocoon are being researched in various fields of health sciences (Rajput and Singh, 2015). Sericin peptides, associated with physical exercise, improve aerobic performance, fat oxidation and increase testosterone (Kim et al., 2013; Shin et al., 2010). Positive results were also reported on the use of sericin for the treatment of burns and wound healing (Aramwit et al., 2013). Although it presents promising characteristics, such as being a biomaterial with potential health applications, its action in neuromuscular regeneration, associated with the therapeutic strategy of swimming exercise, is still unexplored.

Considering the high incidence of PNLs and their effects on skeletal striated musculature, as well as the potential benefits of swimming exercise and sericin, the present study aimed to analyse the effect of sericin associated with the physical exercise of swimming on the phenotype, motor plate and plantar muscle functionality of Wistar rats.

\section{MATERIALS AND METHODS}

Forty Wistar rats weighing $334 \pm 35.2 \mathrm{~g}$ were used, kept in a 12-hr light-dark photoperiod at a temperature of $24^{\circ} \mathrm{C} \pm 1^{\circ} \mathrm{C}$, with water and feed supplied ad libitum. The animals were randomly divided into five experimental groups, with eight rats in each group: control (Ct), lesion (Ls), sericin (Ser), swimming (Swi), and sericin and swimming (Ser+Swi). This study was approved by the Committee on Ethics in the Use of Animals of Universidade Estadual do Oeste do Paraná.

\section{Experimental protocol for sciatic compression}

The animals of the Ls, Ser, Swi, and Ser+Swi groups were weighed and anaesthetised prior to the surgical procedure with an intraperitoneal injection of ketamine hydrochloride (Dopalen) (95 $\mathrm{mg} / \mathrm{kg}$ ) (CEVA, São Paulo, Brazil) and xylazine hydrochloride (Anasedan) $(12 \mathrm{mg} / \mathrm{kg})(\mathrm{CEVA})$. After verification of the state of consciousness of the animal (observed by the absence of motor response to tail clamping and interdigital folds), it was positioned in the ventral decubitus, the thoracic and pelvic limbs being abducted and a trichotomy was performed in the middle third of the right pelvic thigh.

Then, a parallel incision was made into the fibres of the biceps femoris muscle, exposing the sciatic nerve, followed by subsequent compression of the sciatic nerve using haemostatic forceps, for a period of thirty seconds. The pressure generated was standardised by the closing of the forceps on the second tooth of the rack. After pinching, a marking was performed at the lesion site by epineural suture, using 10.0 nylon thread. The Ser and Ser + Nat groups received the application of $100 \mu \mathrm{L}$ of sericin directly on the nerve lesion once during the surgical procedure. After pinching, the nerve was reassembled, a single point skin suture was performed using nylon monofilament yarn, polyvinylpyrrolidone-iodine (Povidine, Geyer, Porto Alegre, Brazil) was applied over the incision and the animals were housed under the same pre-surgical conditions.

\section{Swimming exercise protocol with overload}

Only the Swi and Ser+Swi groups were submitted to physical exercise, although the other groups were exposed to the liquid medium for ten seconds in the same treatment period, so that the stress of the aquatic environment was the same for all groups. All animals were adapted and trained to swim gradually in the fifteen days prior to the nerve injury protocol. Swimming started on the third postoperative day (PO). This was done in an oval-shaped tank of 60-cm depth and capacity of $200 \mathrm{~L}$, and the physical exercise was performed with a water level of 40-cm depth and temperature controlled at $32^{\circ} \mathrm{C}$.

The animals were weighed prior to all sessions to establish the 
load applied during physical exercise, being $10 \%$ of the body mass of the animal, represented by leads that were fixed in the abdomen region. The protocol was followed for 3 weeks, from Monday to Friday, totalling 21 days of treatment, with progressive loading of time: first week after injury $15 \mathrm{~min}$ of swimming, second week 20 min of swimming and third week, 25 min of swimming.

\section{Evaluation of muscle grip strength}

The function of the grip strength test was to verify functionality by means of the muscular strength of the rats that suffered sciatic nerve compression. To perform the evaluation, specific grip strength measurement equipment was used, where the animal was pulled by the back with increasing firmness and allowed to hold with the right pelvic member in a grid connected to a force transducer, until he lost his grip, adapted from Bertelli and Mira (1995).

The left pelvic limb was immobilised manually during the test. The animals were previously adapted and trained in the equipment in the five days before the surgery. The first evaluation (EV1) was done before sciatic nerve compression, to obtain basal values. The evaluations were used to observe the evolution of the lesion and the form of treatment used. In each evaluation, the test was repeated three times and the mean value of the repetitions was used (Coradinia et al., 2015). Other evaluations were carried out during the experiment: 2nd EV, 3rd PO, pretreatment; 3rd EV, 3rd PO, post treatment; 4th EV, 7th PO, post treatment; 5th EV, 8th PO, pretreatment; 6th EV, 14th PO, after treatment; 7th EV, 21st PO, posttreatment; 8th EV, 22nd PO, euthanasia of the animals and collection of the muscles.

\section{Collection of the plantar muscle and histochemical and histoenzymological analyses}

Twenty-four hours after the last treatment session, the animals were weighed and anaesthetised, decapitated in a guillotine and then the plantar muscle was dissected, weighed and measured in length.

For histochemical analysis of NMJs, the proximal part of the muscle was fixed in Karnovsky solution. The evaluation of NMJs was performed by the nonspecific esterase technique (Lehrer and Ornstein, 1959). Morphometric analysis of NMJs was performed on two slides for each animal. In each slide, measurements of the area, larger and smaller diameters of approximately 150 NMJs, were evaluated from microscopic images with a 20x objective.

Fibres and plantar muscle NMJs were photographed and cap- tured under an Olympus Bx60 microscope coupled to an Olympus DP71 camera (Olympus, Tokyo, Japan), with the aid of the DP Controler 3.2.1 276 program. Morphological and morphometric analysis of the fibres and NMJs was conducted using the Image-Pro Plus 6.0 (Media Cybernetics, Rockville, MD, USA) program.

For the histoenzymological study of the muscle fibres, classification according to the proposal of Brooke and Kaiser (1970) was used. The distal portion of the muscle was maintained at room temperature for 30 to $40 \mathrm{~min}$. After this time, the material was covered with neutral talc (JOHNSON, São Paulo, Brazil) for the preservation of the tissue and frozen in liquid nitrogen for $2 \mathrm{~min}$, packed in cryotubes and stored in a Biofreezer at $-80^{\circ} \mathrm{C}$ for further processing. The frozen muscle segments were transferred to a cryostat chamber (LUPETEC CM 2850 Cryostat Microtome, São Carlos, Brazil) at $-20^{\circ} \mathrm{C}$ and held for $30 \mathrm{~min}$ to stabilise the temperature. These segments were then sectioned transversely $(7 \mu \mathrm{m}$ thick) into semiserial sections, which were dewatered, diaphanised, and blades assembled with the aid of Permount (Fisher Scientific, Fair Lawn, NJ, USA). The transverse sections of $7-\mu \mathrm{m}$ thickness were submitted to the Nicotinamide Adenine Dinucleotide Tetrazolium Reductase (NADH-TR) histoenzymological technique for analysis of the oxidative and glycolytic metabolism of muscle fibres, which is based on the ability of the mitochondrial enzyme NADH dehydrogenase to transfer electrons to the colourless, soluble tetrazolium salt and convert it into a formazan compound. This provides an excellent stain of the intermyofibrillar matrix. To demonstrate the activity of NADH-TR, it was shown that muscle fibres of types I (small diameter and intense oxidative activity), IIa (intermediate diameter and moderate oxidative activity), and IIb (large diameter and weak oxidative activity).

Morphometric analyses of muscle fibres were performed by measuring the cross-sectional area and the small diameter of the plantar muscle in approximately 200 fibres per animal. The different types of muscle fibres (I, IIa, and IIb) were also quantified by random selection of two fields $\left(400 \mu \mathrm{m}^{2}\right)$ per animal, from images that were captured with a 20x objective.

\section{Statistical analysis}

The results are presented with values of means and standard deviations. A mixed model analysis of variance (ANOVA) was used to compare the grip strength and unidirectional ANOVA, with post $t$-test (least significant difference), for morphometric comparisons. A significance level of $5 \%$ was considered for all analyses. 
Table 1. Values of plantar muscle grip strength of Wistar rats in grams, for the different moments of evaluation (EV1 to EV8), in the different groups, 22nd postoperative day

\begin{tabular}{|c|c|c|c|c|c|}
\hline Assessment & $C t^{A}$ & $L s^{B}$ & $\mathrm{Ser}^{B}$ & Swi ${ }^{B}$ & Ser+Swi ${ }^{B}$ \\
\hline $\mathrm{EV} 1^{\mathrm{a}}$ & $197.12 \pm 151.8$ & $239.77 \pm 100.7$ & $187.08 \pm 107.1$ & $208.19 \pm 60.3$ & $288.66 \pm 183.4$ \\
\hline $\mathrm{EV} 2^{\mathrm{b}}$ & $257.79 \pm 92.56$ & $0.89 \pm 0.65$ & $1.33 \pm 0.61$ & $0.90 \pm 0.73$ & $1.14 \pm 0.87$ \\
\hline$E 3^{b}$ & $266.79 \pm 118.01$ & $1.22 \pm 0.95$ & $1.0 \pm 0.87$ & $1.04 \pm 0.89$ & $1.04 \pm 0.48$ \\
\hline$E V 4^{b}$ & $199.50 \pm 142.01$ & $0.72 \pm 0.61$ & $0.78 \pm 0.42$ & $0.52 \pm 0.46$ & $0.62 \pm 0.67$ \\
\hline$E V 5^{b}$ & $253.41 \pm 106.35$ & $1.22 \pm 0.71$ & $0.99 \pm 1.06$ & $0.38 \pm 0.40$ & $0.81 \pm 0.60$ \\
\hline$E V 6^{b}$ & $266.62 \pm 242.88$ & $1.22 \pm 1.04$ & $0.91 \pm 0.23$ & $1.76 \pm 1.98$ & $2.38 \pm 3.48$ \\
\hline $\mathrm{EV} 7^{\mathrm{b}}$ & $216.29 \pm 134.50$ & $1.11 \pm 1.00$ & $1.29 \pm 1.55$ & $6.76 \pm 9.53$ & $1.57 \pm 1.88$ \\
\hline$E V 8^{b}$ & $253.29 \pm 90.84$ & $9.11 \pm 20.21$ & $4.54 \pm 6.41$ & $6.47 \pm 4.43$ & $3.52 \pm 3.81$ \\
\hline
\end{tabular}

Values are presented as mean \pm standard deviation. Upper case letters represent differences between groups and lower case between evaluations. Different letters: Statistically different data between groups. Post Student $t$-test.

Ct, control; Ls, lesion; Ser, sericin; Swi, swimming.

Table 2. Values of the macroscopic parameters of the plantar muscle of Wistar rats in the different groups, 22nd postoperative day

\begin{tabular}{lccccc}
\hline Parameter & Ct & Ls & Ser & Swi & Ser+Swi \\
\hline Muscle weight $(\mathrm{g})$ & $0.53 \pm 0.18^{\mathrm{a}}$ & $0.26 \pm 0.10^{\mathrm{b}}$ & $0.29 \pm 0.09^{\mathrm{b}}$ & $0.26 \pm 0.08^{\mathrm{b}}$ & $0.22 \pm 0.10^{\mathrm{b}}$ \\
Length $(\mathrm{mm})$ & $2.79 \pm 0.52^{\mathrm{a}}$ & $2.74 \pm 0.47^{\mathrm{a}}$ & $2.55 \pm 0.34^{\mathrm{a}}$ & $2.58 \pm 0.47^{\mathrm{a}}$ & $2.66 \pm 0.51^{\mathrm{a}}$ \\
\hline
\end{tabular}

Values are presented as mean \pm standard deviation. Different letters: statistically different data between groups. Post Student $t$-test. $P<0.002$.

Ct, control; Ls, lesion; Ser, sericin; Swi, swimming.

\section{RESULTS}

\section{Functional analysis and macroscopic parameters of the plantar muscle}

The analysis of plantar muscle strength and its respective evaluations, as well as weight and length are presented in Tables 1, 2.

Data on the grip strength presented a significant difference $(F[1.6,52.4]=51.6, P<0.001)$, with all groups Ls, Ser, Swi, and $\mathrm{Ser}+$ Swi showing lower values than $\mathrm{Ct}(P<0.001)$ and there was no difference between them.

Regarding the evaluations, the first one was statistically higher in relation to the others $(P<0.001)$ and there was no difference between the other evaluations.

The plantar muscle weight parameter showed a significant difference $(P<0.002)$ in the $C t$ group, in comparison with the other groups. Regarding muscle length $(P=0.862)$, there was no statistically significant difference (Table 2 ).

\section{Morphological and morphometric analysis of NMJs}

The morphological study showed NMJs with a polymorphic shape, being round, oval and elliptical, in all groups studied (Fig. 1). The morphometric analysis did not show significant changes in relation to the area, major and minor diameters, in the groups, $\mathrm{Ct}$, Ls, Ser, Swi, and Ser+Swi (Table 3).
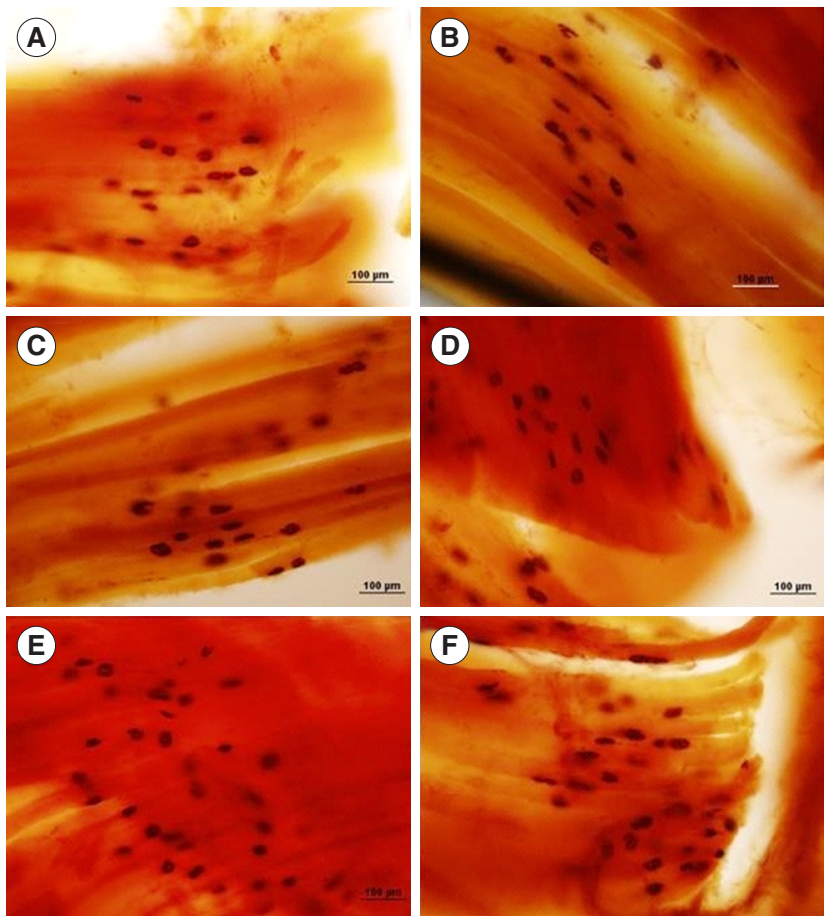

Fig. 1. Photomicrographs of neuromuscular junctions (NMJs) observed in the plantar muscle of Wistar rats, referring to the 22nd postoperative day. Longitudinal section, nonspecific esterase. Control (A), lesion (B), sericin (C), swimming (D), and sericin and swimming (E, F). Observe the NMJ polymorphism. 
Santana AJ, et al. • Sericin and swimming on denervated plantar muscle

Table 3. Morphometry of plantar muscle neuromuscular junctions of Wistar rats in different groups and variables, 22nd postoperative day

\begin{tabular}{lccccc}
\hline Parameter & Ct & Ls & Ser & Swi & Ser+Swi \\
\hline Area & $645.61 \pm 124.5$ & $618.75 \pm 114.1$ & $627.06 \pm 81.38$ & $553.82 \pm 115.7$ & $530.31 \pm 103.5$ \\
Larger diameter & $42.62 \pm 2.37$ & $43.42 \pm 5.12$ & $41.44 \pm 3.63$ & $40.12 \pm 6.01$ & $39.13 \pm 6.37$ \\
Smallest diameter & $18.37 \pm 3.55$ & $18.44 \pm 2.38$ & $18.29 \pm 1.56$ & $16.50 \pm 1.82$ & $16.59 \pm 1.34$ \\
\hline
\end{tabular}

Values are presented as mean \pm standard deviation. Post Student $t$-test. $P>0.05$.

Ct, control; Ls, lesion; Ser, sericin; Swi, swimming.

Table 4. Values found for area of the different types of muscle fibers, I, lla and Ilb, of the plantar muscle of Wistar rats, 22nd postoperative day

\begin{tabular}{lccccc}
\hline Parameter & Ct & Ls & Ser & Swi & Ser+Swi \\
\hline Type I fibre & $1,641.6 \pm 221.4^{\mathrm{a}}$ & $1,237.1 \pm 447.3^{\mathrm{a}}$ & $1,173.4 \pm 137.7^{\mathrm{a}}$ & $1,288.0 \pm 270.0^{\mathrm{a}}$ & $1,302.6 \pm 210.9^{\mathrm{a}}$ \\
Fibre type IIA & $2,313.2 \pm 327.6^{\mathrm{a}}$ & $1,392.1 \pm 389.3^{\mathrm{b}}$ & $1,563.6 \pm 328.3^{\mathrm{b}}$ & $1,411.4 \pm 288.2^{\mathrm{b}}$ & $1,509.9 \pm 345.3^{\mathrm{b}}$ \\
Fibre type IIB & $3,449.0 \pm 498.0^{\mathrm{a}}$ & $1,842.9^{\mathrm{b}} \pm 501.0^{\mathrm{b}}$ & $1,679.7 \pm 283.8^{\mathrm{b}}$ & $1,727.3 \pm 248.3^{\mathrm{b}}$ & $1,944.0 \pm 126.4^{\mathrm{b}}$ \\
\hline
\end{tabular}

Values are presented as mean \pm standard deviation. Different letters: statistically different data between groups. Post Student $t$-test. $P>0.05$.

Ct, control; Ls, lesion; Ser, sericin; Swi, swimming.

Table 5. Values found for morphometric analysis of the smaller diameter of muscle fibres type I, lla and Ilb, of the plantar muscle of Wistar rats, 22nd postoperative day

\begin{tabular}{lccccc}
\hline Smallest diameter & Ct & Ls & Ser & Swi & Ser+Swi \\
\hline Type I fibre & $35.85 \pm 2.28^{\mathrm{a}}$ & $32.57 \pm 5.98^{\mathrm{a}, \mathrm{b}}$ & $30.22 \pm 1.37^{\mathrm{b}, \mathrm{c}}$ & $31.64 \pm 1.97^{\mathrm{b}, \mathrm{c}, \mathrm{d}}$ & $23.71 \pm 2.41$ \\
Fibre type IIA & $45.30 \pm 0.80^{\mathrm{a}}$ & $34.07 \pm 4.63^{\mathrm{b}}$ & $32.71 \pm 2.57^{\mathrm{b}, \mathrm{c}}$ & $33.61 \pm 3.15^{\mathrm{b}, \mathrm{c}, \mathrm{d}}$ & $27.41 \pm 2.69$ \\
Fibre type IIB & $51.93 \pm 4.37^{\mathrm{a}}$ & $39.0 \pm 4.47^{\mathrm{b}}$ & $36.67 \pm 5.38^{\mathrm{b}}$ & $39.22 \pm 4.02^{\mathrm{b}}$ & $34.25 \pm 3.89^{\mathrm{b}}$ \\
\hline
\end{tabular}

Values are presented as mean \pm standard deviation. Different letters: statistically different data between groups. Post Student $t$-test. $P>0.05$.

Ct, control; Ls, lesion; Ser, sericin; Swi, swimming.

\section{Histoenzymological analysis of the plantar muscle}

There was a statistically significant difference in the area of type I fibres in the Ct group compared with the other groups, with no significant difference between the other groups. There was also a significant difference in the area of the fibres of type IIa and IIb, in relation to the group $\mathrm{Ct}$ compared with the other groups, with no difference between them (Table 4). As for the smaller diameter of the fibres, there was a statistically significant difference in the types of fibres (I, IIa, and IIb) between the groups, according to Table 5. Regarding muscle fibre counts, there was a statistically significant difference for the type I fibres of the Ct group in relation to the other groups, and the Ls group also presented a significant difference in relation to the Ser, Swi, and Ser+Swi groups, with a reduction in the counting of fibres in $28 \%, 31 \%$, and $29 \%$, respectively, which did not differ between the other groups. The counts of type IIa and IIb fibres did not present a statistically significant difference between the groups (Table 6) (Fig. 2).

\section{DISCUSSION}

The sciatic nerve compression model performed in the present study reproduces an axonotmosis-type lesion, which is characterised by interruption of the nerve stimulus, producing alterations in muscle morphology, directed towards atrophy, the main effect being a reduction of the fibre area and diameter, with consequent reduction of force. The histoenzymological analysis of the present study demonstrated that the nerve injury could alter the plantar muscle morphology of all injured groups, when compared to the control group, although not to the point of altering the specific characteristics of the different types of fibres.

Zhong et al. (2007) described reduction of muscle mass and muscle fibre size of the medial (GM) and anterior tibial gastrocnemius (TA) muscles in a peripheral nerve injury model. Similar changes were also described by Patterson and Stephenson (2006) in the soleus and extensor digitorum skeletal muscles in a model of ischial denervation of Long-Evans rats.

The morphometry of the present study demonstrated differences in the control group in relation to the other groups for the area of type IIa and IIb fibres, but did not reveal differences between the treated groups. As for the area of type I fibres, there was no significant difference between the groups. This demonstrates that the nerve injury produced did not significantly affect the area of 
Table 6. Counting of the different types of muscle fibres I, lla, and IIb, of the plantar muscle of Wistar rats, 22nd postoperative day

\begin{tabular}{lccccc}
\hline Parameter & Ct & Ls & Ser & Swi & Ser+Swi \\
\hline Type I fibre & $92.20 \pm 11.60^{\mathrm{a}, \mathrm{b}, \mathrm{c}}$ & $105.50 \pm 41.34^{\mathrm{b}, \mathrm{c}}$ & $147.20 \pm 30.86^{\mathrm{c}}$ & $154.40 \pm 17.15^{\mathrm{c}}$ & $149.20 \pm 16.81^{\mathrm{c}}$ \\
Fibre type IIA & $61.60 \pm 8.26^{\mathrm{a}}$ & $65.75 \pm 27.58^{\mathrm{a}}$ & $70.40 \pm 16.36^{\mathrm{a}}$ & $61.40 \pm 15.72^{\mathrm{a}}$ & $60.60 \pm 11.21^{\mathrm{a}}$ \\
Fibre type IIB & $73.0 \pm 13.19^{\mathrm{a}}$ & $63.25 \pm 16.15^{\mathrm{a}}$ & $74.60 \pm 12.21^{\mathrm{a}}$ & $57.40 \pm 16.08^{\mathrm{a}}$ & $69.80 \pm 7.94^{\mathrm{a}}$ \\
\hline
\end{tabular}

Values are presented as mean \pm standard deviation. Different letters: statistically different data between groups. Post Student $t$-test. $P>0.05$.

Ct, control; Ls, lesion; Ser, sericin; Swi, swimming.
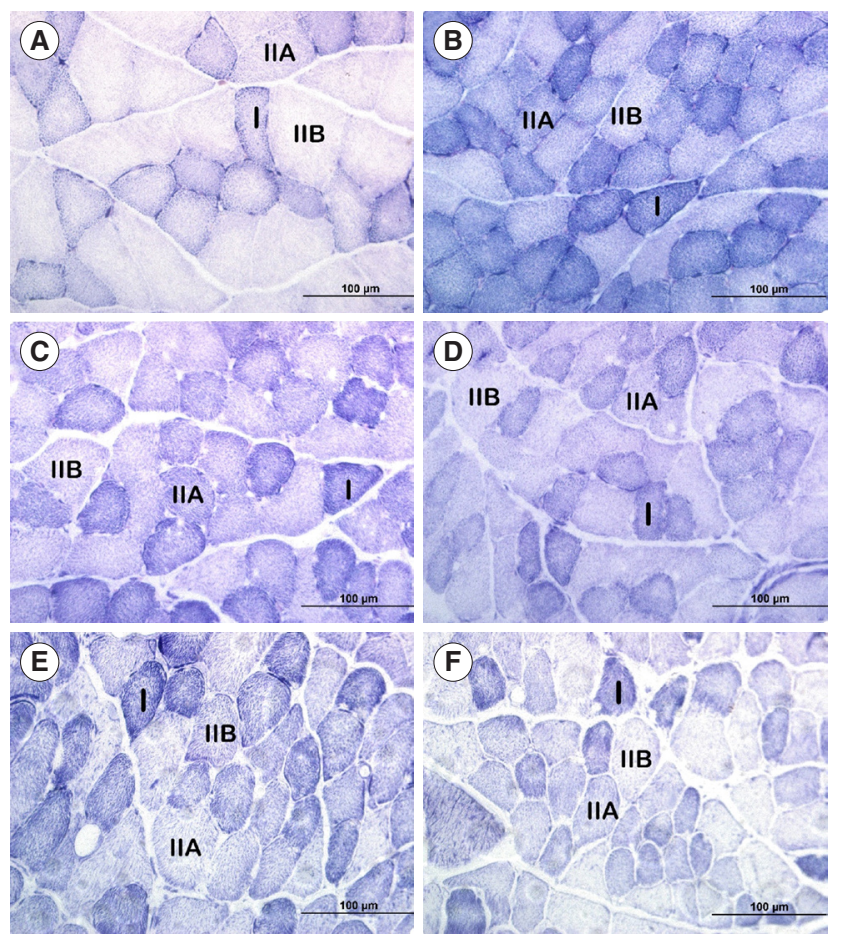

Fig. 2. Photomicrographs of the plantar muscle of Wistar rats, 22nd postoperative day. Cross section, NADH-TR (Nicotinamide Adenine Dinucleotide Tetrazolium Reductase) reaction. Muscle fibres of types I, Ila, and Ilb. Control (A), lesion $(B)$, sericin $(C)$, swimming (D), and sericin and swimming $(E, F)$.

the plantar muscle fibres, different from that observed in other muscles (Patterson and Stephenson, 2006; Zhong et al., 2007). Although the Ls group presented a reduction in the smaller diameter of the type I and IIa fibres in relation to the sericin group and swimming exercise group (Ser+Swi).

In analysing muscle mass, Tanaka et al. (2005) describes that there was no alteration of muscle mass of the soleus muscle, in a model of denervation of the tibial nerve of Wistar rats. Similar results were described by Snow et al. (2005) in the soleus muscle of Sprague Dawley rats, in a model of peripheral neuropathy. However, the weight of the plantar muscle in the present study presented a statistically significant difference in relation to the other groups, demonstrating that there was a decrease in muscle mass as a consequence of PNL, confirming that neuromuscular activity is determinant in maintaining muscle properties. As for the other groups, there were no differences recorded. Although muscle weight in the control group was statistically significant to the detriment of all other groups that suffered injury, the parameter length of the muscle was not altered in any of the groups, showing that although the nerve injury caused a reduction in muscle weight, this was not intense enough to produce longitudinal changes of the musculature.

In our research, the hypothesis that sericin associated with swimming exercise could be an important factor in the recovery from paresis did not materialise, even considering sericin's characteristics in cell proliferation and division (Aramwit et al., 2013; Shin et al., 2010), as well as the action of exercise in reducing protein degradation (Lee et al., 2012), possible endogenous opioid-mediated analgesia (which could reduce limb functional deficit) (Bertolini et al., 2012), as well as water properties such as: increased peripheral circulation, improved oxygen and nutrient supply, increased blood and lymphatic return, decreased oedema due to the action of hydrostatic pressure and reduced sensitivity of the nerve terminals (Medeiros et al., 2004).

The results of this study show that in the first evaluation performed preoperatively, the animals presented greater strength when compared with the others, therefore pertinent with normality patterns, there was no difference between the other evaluations and groups. The reduction of force may be related to hypernociception generated by nerve compression, which produces muscle inhibition (Yahia et al., 2010). In addition, studies have shown that treatment with swimming exercise may not be able to reduce the pain of rats submitted to sciatica (Gaffuri et al., 2011).

The contractile activity of skeletal muscle is regulated by the central nervous system, through the transmission of action potentials from motor neurons to muscle fibres. Both the presynaptic motor axon and postsynaptic skeletal muscle fibres are highly specialised in NMJs, thus guaranteeing an efficient transmission of the action potential (Kwon and Yoon, 2017). They are subject to remodelling due to several factors: age, denervation, ageing (Gon- 
zalez-Freire et al., 2014), muscle fibre type, activity and hormonal status (Deschenes et al., 2015), some congenital diseases such as Myasthenia Gravis and Lambert-Eaton syndrome, besides several forms of intoxication, one of them botulism (Kwon and Yoon, 2017).

However, both the morphological analysis and the morphometry of the NMJs measured in the present study did not present significant differences. The polymorphism of NMJs can be observed in the morphological analysis, although there was no significant difference for the morphometric parameters of the area, major and minor diameters. Although the nerve injury was characterised, it was not able to alter the morphology of the NMJs, perhaps due to the regenerative capacity of the peripheral nervous system, considering the time elapsed - 22 days postoperatively. However, Torrejais et al. (2009) analysing the morphometry and morphology of diaphragm NMJs of denervated novergicus rats at different periods, observed that after denervation the NMJs presented a smaller and elongated appearance, having less visible contours. Changes in NMJ morphometry were also described by Liu et al. (2016) in a denervation model of the anterior tibial nerve of Sprague Dawley rats.

Patterson and Stephenson (2006) found changes in the muscle fibre phenotype of the Long-Evans rat soleus muscle, with a reduction of the slow isoform MHCI, $77 \%$ to $61 \%$, a concomitant increase of the rapid isoform MHCIIA from 23\% to 39\%, as well as changes in the morphometry of different types of fibres in a sciatic nerve injury model.

The results of the present study verified that the nerve injury altered the myosin heavy chain isoform, with reduction of the slow isoform, considering that the plantar muscle has a rapid myosin heavy chain phenotype predominance (Chaillou et al., 2013). These results are corroborated by the literature which demonstrates that phasic muscles when denervated tend to become (slow) tonic muscles (Patterson and Stephenson, 2006; Rowan et al., 2012; Snow et al., 2005; Zhong et al., 2007). The counting of muscle fibres showed a significant difference for type I fibres in the $\mathrm{Ct}$ group in relation to the other groups, and the Ls group also presented a significant difference in relation to the Ser, Swi, and Ser+Swi groups, with a reduction in the percentage of fibres in $28 \%, 31 \%$, and $29 \%$, respectively, with no difference between the other groups studied.

Although physical exercise affects the organism as a whole, the main positive impacts are the adaptations that occur in the skeletal muscle. Like denervation, physical exercise is capable of causing changes in the muscle fibre phenotype, specifically, aerobic exercise promotes transformation in fibre type in the fast to slow (type IIb/IIx to IIa) in muscles with normal patterns of innervation (Yan et al., 2011). However, swimming exercise in the present study was not able to promote transformation in the plantar muscle phenotype. It is assumed that nerve damage has supplanted the ability of swimming exercise as well as its association with sericin in transforming the contractile properties of muscle.

Similar results were also described by Tanaka et al. (2005) in a model of partial denervation of the anterior tibial nerve, who found that treadmill aerobic exercise was not efficient in promoting phenotypic transformation in the soleus muscle of Wistar rats. Kim et al. (2015) also found that aerobic ladder exercise for 8 weeks was unable to change the myosin heavy chain ratios in the medial gastrocnemius muscle of Zucker rats.

In this study, axonotmosis-type nerve damage was not sufficient to modify the morphology of NMJs, as well as alter skeletal muscle functionality. The association of sericin with swimming exercise in the acute phase was not an effective therapeutic modality to transform the contractile properties of the plantar muscle, although the nerve injury did.

The association of sericin with swimming exercise was not efficient in terms of recovery of grip strength and alteration of contractile muscle properties, altered by experimental axonotmosis.

\section{CONFLICT OF INTEREST}

No potential conflict of interest relevant to this article was reported.

\section{REFERENCES}

Aramwit P, Palapinyo S, Srichana T, Chottanapund S, Muangman P. Silk sericin ameliorates wound healing and its clinical efficacy in burn wounds. Arch Dermatol Res 2013;305:585-594.

Bertelli JA, Mira JC. The grasping test: a simple behavioral method for objective quantitative assessment of peripheral nerve regeneration in the rat. J Neurosci Methods 1995;58:151-155.

Bertolini GR, Rosa CT, Silva LI, Meireles A, Rocha BP. Use of resistance exercise as a factor antagonized by naloxone of analgesia in acute knee synovitis in Wistar rats. Rev Bras Med Esporte 2012;18:126-129.

Brooke MH, Kaiser KK. Muscle fiber types: how many and what kind? Arch Neurol 1970;23:369-379.

Chaillou T, Koulmann N, Meunier A, Malgoyre A, Serrurier B, Beaudry $\mathrm{M}$, Bigard X. Effect of hypoxia exposure on the phenotypic adaptation in remodelling skeletal muscle submitted to functional overload. Acta 
Physiol (Oxf) 2013;209:272-282.

Coradinia JG, Kakihata CM, Kunz RI, Errero TK, Bonfleur ML, Bertolini GR. Evaluation of grip strength in normal and obese Wistar rats submitted to swimming with overload after median nerve compression. Rev Bras Reumatol 2015;55:43-47.

da Silva CK, Camargo EA. Mechanisms involved in the regeneration of peripheral nerve injuries. Rev Saúde Pesqui 2010;3:93-98.

Deschenes MR, Roby MA, Glass EK. Aging influences adaptations of the neuromuscular junction to endurance training. Neuroscience 2011; 190:56-66.

Deschenes MR, Sherman EG, Roby MA, Glass EK, Harris MB. Effect of resistance training on neuromuscular junctions of young and aged muscles featuring different recruitment patterns. J Neurosci Res 2015; 93:504-513.

Gaffuri J, Meireles A, Rocha BP, Rosa CT, Artifon EL, Silva LI, Moreira NB, Bertolini GR. Physical exercise assessment as an analgesia factor in a sciatica experimental model. Rev Bras Med Esporte 2011;17:115118.

Geuna S. The sciatic nerve injury model in pre-clinical research. J Neurosci Methods 2015;243:39-46.

Gonzalez-Freire M, de Cabo R, Studenski SA, Ferrucci L. The neuromuscular junction: aging at the crossroad between nerves and muscle. Front Aging Neurosci 2014;6:208.

Kääriäinen M, Kauhanen S. Skeletal muscle injury and repair: the effect of disuse and denervation on muscle and clinical relevance in pedicled and free muscle flaps. J Reconstr Microsurg 2012;28:581-587.

Kim J, Hwang H, Yun HY, Kim B, Lee CH, Suh H, Lim K. Silk peptide intake increases fat oxidation at rest in exercised mice. J Nutr Sci Vitaminol (Tokyo) 2013;59:250-255.

Kim JY, Choi MJ, So B, Kim HJ, Seong JK, Song W. The preventive effects of 8 weeks of resistance training on glucose tolerance and muscle fiber type composition in zucker rats. Diabetes Metab J 2015;39:424-433.

Kwon YN, Yoon SS. Sarcopenia: neurological point of view. J Bone Metab 2017;24:83-89.

Lee Y, Kim JH, Hong Y, Lee SR, Chang KT, Hong Y. Prophylactic effects of swimming exercise on autophagy-induced muscle atrophy in diabetic rats. Lab Anim Res 2012;28:171-179.

Lehrer GM, Ornstein L. A diazo coupling method for the electron microscopic localization of cholinesterase. J Biophys Biochem Cytol 1959;6: 399-406.

Li R, Liu Z, Pan Y, Chen L, Zhang Z, Lu L. Peripheral nerve injuries treatment: a systematic review. Cell Biochem Biophys 2014;68:449-454.

Liu L, Xie F, Wei K, Hao XC, Li P, Cao J, Min S. Sepsis induced denerva- tion-like changes at the neuromuscular junction. J Surg Res 2016;200: 523-532.

Malysz T, Ilha J, Severo do Nascimento P, Faccioni-Heuser MC, De Angelis K, D'agord Schaan B, Achaval M. Exercise training improves the soleus muscle morphology in experimental diabetic nerve regeneration. Muscle Nerve 2011;44:571-582.

Medeiros A, Oliveira EM, Gianolla R, Casarini DE, Negrão CE, Brum PC. Swimming training increases cardiac vagal activity and induces cardiac hypertrophy in rats. Braz J Med Biol Res 2004;37:1909-1917.

Patterson MF, Stephenson GM, Stephenson DG. Denervation produces different single fiber phenotypes in fast- and slow-twitch hindlimb muscles of the rat. Am J Physiol Cell Physiol 2006;291:C518-C528.

Rajput SK, Singh MK. Sericin - a unique biomaterial. IOSR J Polym Text Eng 2015;2:29-35.

Rowan SL, Rygiel K, Purves-Smith FM, Solbak NM, Turnbull DM, Hepple RT. Denervation causes fiber atrophy and myosin heavy chain co-expression in senescent skeletal muscle. PLoS One 2012;7:e29082.

Shin S, Yeon S, Park D, Oh J, Kang H, Kim S, Joo SS, Lim WT, Lee JY, Choi KC, Kim KY, Kim SU, Kim JC, Kim YB. Silk amino acids improve physical stamina and male reproductive function of mice. Biol Pharm Bull 2010;33:273-278.

Snow LM, Sanchez OA, McLoon LK, Serfass RC, Thompson LV. Effect of endurance exercise on myosin heavy chain isoform expression in diabetic rats with peripheral neuropathy. Am J Phys Med Rehabil 2005; 84:770-779.

Tanaka S, Tsubaki A, Tachino K. Effect of exercise training after partial denervation in rat soleus muscles. J Phys Ther Sci 2005;17:97-101.

Torrejais MM, Soares JC, Matheus SM, Mello JM, Francia-Farje LA, Vicente EJ. Morphometric and morphological analysis of neuromuscular junction alterations in the denervated rat diaphragm. Int J Morphol 2009;27:1235-1242.

Wu H, Xiong WC, Mei L. To build a synapse: signaling pathways in neuromuscular junction assembly. Development 2010;137:1017-1033.

Yahia A, Ghroubi S, Kharrat O, Jribi S, Elleuch M, Elleuch MH. A study of isokinetic trunk and knee muscle strength in patients with chronic sciatica. Ann Phys Rehabil Med 2010;53:239-249.

Yan Z, Okutsu M, Akhtar YN, Lira VA. Regulation of exercise-induced fiber type transformation, mitochondrial biogenesis, and angiogenesis in skeletal muscle. J Appl Physiol (1985) 2011;110:264-274.

Zhong H, Roy RR, Woo J, Kim JA, Edgerton VR. Differential modulation of myosin heavy chain phenotype in an inactive extensor and flexor muscle of adult rats. J Anat 2007;210:19-31. 